\title{
Was hat Routine mit Jobpolarisierung zu tun?
}

\section{Brigitte Tschudi}

\section{Relevanz}

Kommt der Mittelstand unter Druck? Innovation und Globalisierung steigern Arbeitsproduktivität, Löhne und Beschäftigung. Gerade die innovativsten Unternehmen brauchen Zugang zum Weltmarkt, um stärker zu wachsen und mehr Jobs zu schaffen. Doch die Vorteile sind ungleich verteilt. Dabei sind Innovation und Globalisierung nicht nur ein Gewinn für die hoch qualifizierten und eine Gefahr für die gering ausgebildeten Arbeitnehmer. Die Automatisierung ist dort erfolgreich, wo viele repetitive Routinetätigkeiten anfallen. Berufe mit viel Routine sind gerade in den mittleren Einkommensgruppen stark vertreten. Automatisierung könnte den Mittelstand ausdünnen und zu einer Polarisierung der Arbeitswelt am unteren und oberen Einkommensende führen. Der Sozialstaat kann die Einkommensund Beschäftigungsrisiken der Arbeitenden wenigstens teilweise ausgleichen. Eine investive Politik wird jedoch vorbeugend an der Ausbildung und am lebenslangen Lernen ansetzen, damit möglichst viele den Routinetätigkeiten ausweichen und mehr kreative und höher qualifizierte Tätigkeiten übernehmen können.

Christian Keuschnigg

B. Tschudi $(\bowtie)$

Universität St. Gallen, St. Gallen, Schweiz

E-Mail: brigitte.tschudi@student.unisg.ch 


\section{Quelle}

Der nachfolgende Text ist eine Zusammenfassung von: Goos, Maarten, Alan Manning und Anna Salomons (2014), Explaining Job Polarization: Routine-Biased Technological Change and Offshoring, American Economic Review 104(8), 2509-2526.

Technologischer Wandel und Globalisierung gelten schon lange als Gründe für steigende Ungleichheit. Nach weit verbreiteter Ansicht steigert der technologische Fortschritt die Produktivität von qualifizierten Arbeitnehmern und verbessert ihre Aussichten auf gute Jobs und höhere Löhne, während unqualifizierte Arbeit wenig davon hat oder sogar bedroht ist. Neuere Forschungsergebnisse zeigen dagegen, dass die Beschäftigung seit einiger Zeit vor allem in den durchschnittlich qualifizierten Berufen eingebrochen ist. Jobs mit hohen und tiefen Qualifikationen sind eher stärker gefragt. Experten sprechen von einer Polarisierung der Arbeitswelt. Während die Jobpolarisierung in einigen wichtigen Ländern gut dokumentiert ist, sind die Ursachen dafür noch weitestgehend unbekannt.

Genau hier setzt die Studie von Goos, Manning und Salomons aus dem Jahr 2014 an. Die drei Forscher sind der Ansicht, dass technologischer Wandel und Globalisierung auch für die Polarisierung der Beschäftigung verantwortlich sind. Die ausschlaggebende Ursache dafür, wer vom technologischen Fortschritt profitiert und wer nicht, sehen die Forscher nicht im Qualifikationsniveau an sich, sondern in der Routineintensität eines Berufs. Der technologische Wandel führe dazu, dass Arbeitskräfte, die überwiegend Routinetätigkeiten ausführen, durch Maschinen ersetzt würden. Globalisierung führe dagegen zur Verlagerung von Arbeit ins Ausland. Das Ergebnis ist dasselbe, weniger Jobs und geringe Aussichten auf bessere Löhne. Anscheinend wirken beide Kräfte besonders stark auf Berufe mit mittleren Qualifikationen ein und führen zu einer Ausdünnung des Mittelstands, also einer Polarisierung bei hoch und gering qualifizierter Arbeit. Die Daten zeigen, dass vorwiegend der technologische Wandel und weniger die Globalisierung zur Polarisierung der Arbeit beigetragen hat. Dies betrifft die Beschäftigung sowohl innerhalb einer Branche als auch zwischen Branchen.

Die Forscher vergleichen die Entwicklung der Beschäftigungsanteile verschiedener Berufe in 16 westeuropäischen Staaten zwischen 1993 und 2010. Die Beschäftigung von hoch bezahlten Berufen, wie z. B. von Ingenieuren, hat am stärksten zugenommen. Ihr Anteil an der Gesamtbeschäftigung ist von 1993 bis 2010 um durchschnittlich 5,6 Prozentpunkte gestiegen. Der Anteil gering bezahlter Berufe an der Gesamtbeschäftigung stieg im selben Zeitraum ebenfalls, und zwar um rund 3,7 Prozentpunkte im Durchschnitt. In dieser Gruppe konnte die Beschäftigung in Verpflegungs- und Reinigungsberufen zulegen, während sie 
im Verkauf sowie im Baugewerbe und in der Produktion rückläufig war. Berufe mit mittlerem Einkommen, wie z. B. Büroangestellte oder Handwerker, haben dagegen sehr stark an Bedeutung verloren. Von 1993 bis 2010 ging ihr Beschäftigungsanteil um durchschnittlich 9,3 Prozentpunkte zurück.

Während die Beschäftigungsanteile von hoch und niedrig bezahlten Berufen von 1993 bis 2010 merklich zugenommen haben, ist der Beschäftigungsanteil von durchschnittlich bezahlten Berufen deutlich geschrumpft.

Abb. 1 zeigt große Unterschiede zwischen den untersuchten Staaten. In Österreich und Italien wuchs der Beschäftigungsanteil der gering bezahlten Berufe sogar stärker als jener der hoch bezahlten, in allen anderen Staaten war es umgekehrt. Allen Staaten gemeinsam ist jedoch die Ausdünnung des Mittelstands, also der starke Rückgang des Anteils von Arbeitskräften mit durchschnittlichem Einkommen.

Die Abb. 1 zeigt die Beschäftigungsveränderung von 1993 bis 2010 in vier niedrig, neun durchschnittlich und acht hoch bezahlten Berufen.

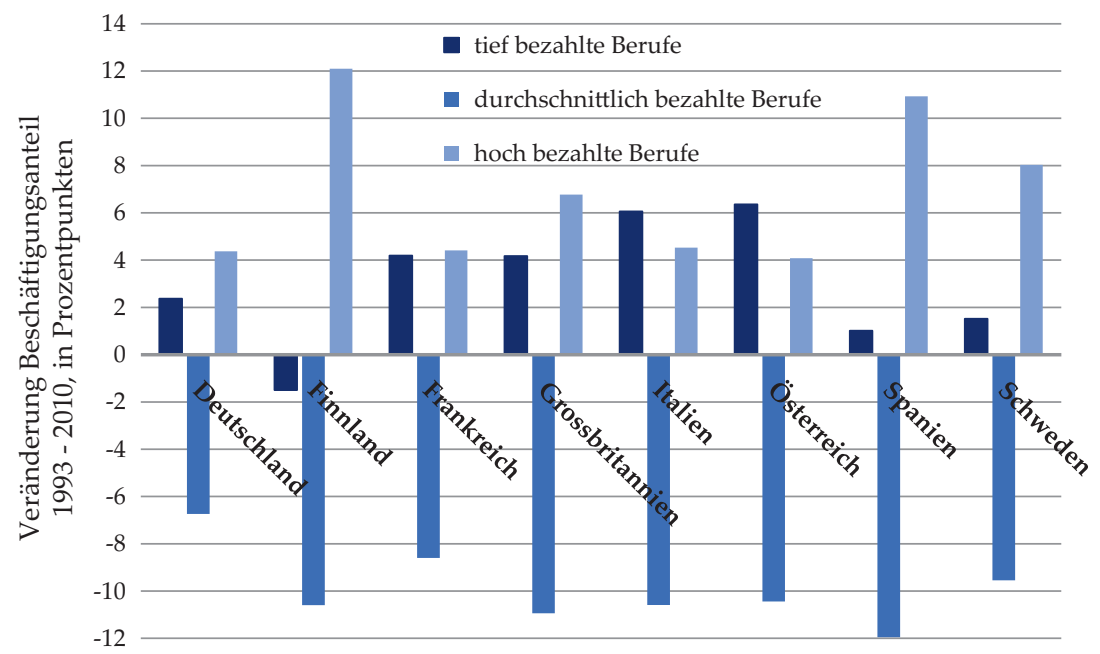

Abb. 1 Beschäftigungsveränderung 1993-2010 in ausgewählten Staaten. (Eigene Darstellung nach Goos u. a., 2014, S. 2515, Tab. 2) 
Die Wissenschaftler untersuchen Daten aus der sektoralen Arbeitskräfteerhebung der EU und der OECD. Die Routineintensität einer Aufgabe messen sie anhand eines gebräuchlichen Index, wonach der Beruf des Büroangestellten den stärksten Routineanteil aufweist. Am wenigsten Routine hat der Geschäftsführer eines kleinen Unternehmens, der offensichtlich alles können muss. Die Forscher verwenden auch ein $\mathrm{Ma} ß$ für den Grad, wie stark eine Tätigkeit ins Ausland verlagert werden kann. Nach diesem Index ist es am leichtesten, die Tätigkeit eines Maschinenführers ins Ausland zu verlagern. Am schwierigsten ist die Arbeit eines Logistikers auszulagern. Die Korrelation zwischen diesen beiden Jobcharakteristiken beträgt 0,46 . Ein Job mit hohem Routineanteil kann nicht nur leichter automatisiert, sondern auch eher in das Ausland verlagert werden.

Die Forscher finden, dass weniger die Globalisierung, sondern vielmehr der technologische Fortschritt für die Polarisierung der Arbeitswelt verantwortlich ist. Die zunehmende Automatisierung routineintensiver Tätigkeiten bremst vor allem die Beschäftigung in den Berufen mit mittleren Einkommen im Vergleich zu gering und hoch bezahlten Jobs. Nimmt die Routineintensität eines Berufs um eine Standardabweichung $\mathrm{zu}$ - das entspricht dem Unterschied zwischen der Routineintensität eines Arztes und eines Verkäufers -, so sinkt das Beschäftigungswachstum dieses Berufs um 0,9 Prozentpunkte. Der Grad einer möglichen Verlagerung von Jobs in das Ausland hat hingegen keinen statistisch signifikanten Einfluss auf das Beschäftigungswachstum.

Indem sie die Berufe nach ihrer Routineintensität und ihrer Anfälligkeit für eine Verlagerung in das Ausland klassifizieren, können die Forscher einen sehr großen Teil der tatsächlich beobachteten Beschäftigungsentwicklung erklären, nämlich $79 \%$ bei hoch bezahlten, $74 \%$ bei durchschnittlich und $66 \%$ bei niedrig bezahlten Berufen.

Für den Rückgang der Beschäftigung in routineintensiven Berufen mit mittleren Einkommen ist vor allem der technologische Fortschritt verantwortlich. Eine Zunahme der Routineintensität um eine Standardabweichung verlangsamt das Beschäftigungswachstum um 0,9 Prozentpunkte.

Die Veränderung der Beschäftigung eines Berufs kann sich dabei aufgrund von Verschiebungen innerhalb und zwischen den Branchen ergeben. Der Beschäftigungsanteil eines Berufes kann sich wegen Verschiebungen der Arbeitsnachfrage von durchschnittlich zu hoch bzw. niedrig bezahlten Tätigkeiten innerhalb einer Branche verändern (intraindustrielle Komponente). Außerdem kann der Beschäftigungsanteil eines Berufs zunehmen, wenn Branchen mit hohem Bedarf nach 
diesem Beruf expandieren und andere Branchen mit geringem Bedarf schrumpfen, oder eben abnehmen, wenn sich die Branchen gerade umgekehrt verändern (interindustrielle Komponente). Die Ergebnisse zeigen, dass beide Komponenten in ähnlichem Umfang zur Gesamtveränderung beitragen. Bei hoch und durchschnittlich bezahlten Berufen sind die Beschäftigungsverschiebungen innerhalb der Branche bedeutender, während bei niedrig bezahlten Berufen die Verlagerung zwischen den Branchen eher wichtiger ist.

Die intraindustrielle und die interindustrielle Komponente tragen in etwa zu gleichen Teilen zur beobachteten Beschäftigungsveränderung der Berufe bei.

Die Studie zeigt, dass vor allem der technologische Fortschritt und weniger die Globalisierung die Ausdünnung des Mittelstands und die Polarisierung der Arbeit prägt. Automatisierung und der häufigere Einsatz von Robotern ersetzen zunehmend Routinetätigkeiten. Dadurch nehmen die Anteile hoch und niedrig bezahlter Tätigkeiten an der Beschäftigung zu, während der Anteil routineintensiver Berufe mit mittleren Einkommen zurückgeht.

Open Access Dieses Kapitel wird unter der Creative Commons Namensnennung 4.0 International Lizenz (http://creativecommons.org/licenses/by/4.0/deed.de) veröffentlicht, welche die Nutzung, Vervielfältigung, Bearbeitung, Verbreitung und Wiedergabe in jeglichem Medium und Format erlaubt, sofern Sie den/die ursprünglichen Autor(en) und die Quelle ordnungsgemäß nennen, einen Link zur Creative Commons Lizenz beifügen und angeben, ob Änderungen vorgenommen wurden.

Die in diesem Kapitel enthaltenen Bilder und sonstiges Drittmaterial unterliegen ebenfalls der genannten Creative Commons Lizenz, sofern sich aus der Abbildungslegende nichts anderes ergibt. Sofern das betreffende Material nicht unter der genannten Creative Commons Lizenz steht und die betreffende Handlung nicht nach gesetzlichen Vorschriften erlaubt ist, ist für die oben aufgeführten Weiterverwendungen des Materials die Einwilligung des jeweiligen Rechteinhabers einzuholen.

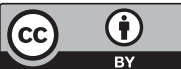

